\title{
Gravitational noise is asymmetric in the time domain and exists permanently
}

\author{
Alexander V. Kramarenko ${ }^{a}$, Andrey V. Kramarenko, ${ }^{b, *}$, Oksana O. Savenko ${ }^{c}$ \\ ${ }^{a}$ TREDEX Company Ltd., PO box 11515, Kharkiv, Ukraine 61001 \\ ${ }^{b}$ General and Inorganic Chemistry Department, National Technical University "KhPI", 2 Kyrpychova str., Kharkiv, \\ Ukraine 61002 \\ ${ }^{c}$ School of Physics and Technology, V. N. Karazin Kharkiv National University, 4 Svobody Sq., Kharkiv, Ukraine 61022
}

\begin{abstract}
Analyzing the records of gravitational observatories of the LIGO project [1] we have found a specific asymmetry in the time domain, inherent only to the signals of their gravitational detectors. Its comparison with various periodic signals, Gaussian and non-Gaussian noises made it possible to draw a preliminary conclusion that the noise of gravitational detectors is a unique mixture of signals.

We have developed our own system of filtering gravitational signals, which uses only linear time-invariant elements [2] . All processing is performed exclusively in the time domain. Detection and recognition of gravitational wave signals were carried out using a specialized Pearson [ $\underline{3}$ ] correlation analyzer.

It has been revealed that the detectors' noises include a significant $(-6 \mathrm{~dB})$ component inherent to the reliably detected gravitational waves. This result allows one to reasonably assume that the gravitational noise is largely due to the merging processes of massive astronomical objects. Since the specific noise signal is registered by detectors continuously, the field of gravitational oscillations of sub-kilohertz range can be considered as detected.

A method of analysis has been developed to estimate the contribution of the gravitational noise component to the total signal energy. Its advantage can be considered a low requirement for computing power: signal processing is provided in real-time using any PC.

With the help of the proposed method, it will be possible not only to begin the radiofrequency estimation of the magnitude of gravitational disturbances, which follows from the recent publications [4] but also, possibly, to construct a map of the gravitational noise of the sky.

The hyperlinks to the data files we have been working with, also as the result files are in the paper. We also can provide the source code of our program upon a reasonable request.
\end{abstract}

\section{Introduction}

By now, all the gravitational waves detected by the LIGO [1] project obsevatories and having instantaneous amplitude $h(t)=h_{0} \cos \phi(t)=h_{0} \cos \left(2 \pi f t+\pi \dot{f} t^{2}-\phi_{0}\right)$ are the pulse signals with combined frequency-amplitude modulation, smooth rise and rapid fall. The linear frequency modulated (LFM) radar signals [ [ $]$ have a similar asymmetry in time, which is realized only in the area of instantaneous frequencies, but the pulse amplitude does not change significantly.

The optimal detector of a signal with such a specific form would be a convolution with its time-inverted copy [] ]. At the same time the convolution with the noninverted copy will give a much smaller response, which is not concentrated in time. This phenomenon is caused by the asymmetry of pulses.

\footnotetext{
* Principal corresponding author

Email addresses: tredexcompany37@gmail.com (Alexander V. Kramarenko), andrii.kramarenko@khpi.edu.ua (Andrey V. Kramarenko), xana.savenko@gmail.com (Oksana O. Savenko)
} 
The question about the contribution of gravitational waves due to the merging of rotating masses to the total noise structure has been discussed many times. As far as we know [7], the discussion has not yet gone beyond hypotheses, and obtaining experimental data would be useful. Separately, we should mention the work [ $\underline{8}]$, the authors of which proposed a method for detecting stellar mass merger events based on small differences between the observed noise and the Gaussian noise.

However, the Gaussian criteria of noise in no way forbids it to be symmetric or asymmetric in time. As an example, let us take a sufficiently large realization of ideal white Gaussian noise and divide it into a set of equal fragments. After spectral analysis, we calculate the median frequency of the spectrum of each fragment. Let us assemble a new realization from the fragments, where the first fragments will have low frequencies of the spectrum, the second fragments will have middle frequencies, the third fragments will have high frequencies, and so on cyclically.

The resulting realization will fully correspond to all the criteria of the Gaussian white noise (GWN), as the original one. But the spectrum analyzer when shifting from left to right along the new realization (using a sliding window equal to the length of the fragment), will generate not a random, but a cyclically repeated sequence of median frequencies. And at the reverse pass the sequence will repeat in the reverse order. In other words, the GWN realization constructed in this way will not be symmetric (invariant) in time. But the distribution function and spectrum of the new realization will not differ from the original ones.

Therefore, the search for the noninvariance of gravitational noise over time seems to us more promising than the analysis of the distribution function and spectra, because the signals of rotation/merging of stellar masses are asymmetric in the time domain.

\section{Signal processing methods}

\subsection{The raw signal filtration.}

In order to answer the question of the contribution of gravitational wave energy to the total noise energy it is necessary to check: is there a characteristic for gravitational waves asymmetry in the detector's output signal in the period when these events, i. e. waves, are not fixed. This problem must be solved only in the time domain (without transition to the frequency domain), so, spectral whitening will have to be excluded. Moreover, the filtering should provide a linear phase-frequency response of the whole system, because any "loop" signal attenuation, typical for high order IIR filters, will distort the results []].

The nonrecursive filters needed to solve the described problem were constructed using a sliding window having weight coefficients of the form:

$$
W_{i}=-\cos \frac{2 \pi i}{N-1} \cdot \sin ^{2} \frac{\pi i}{N-1}
$$

where $i \in[0, N-1]$ is the number of the readout inside the window of length $N$ readouts.

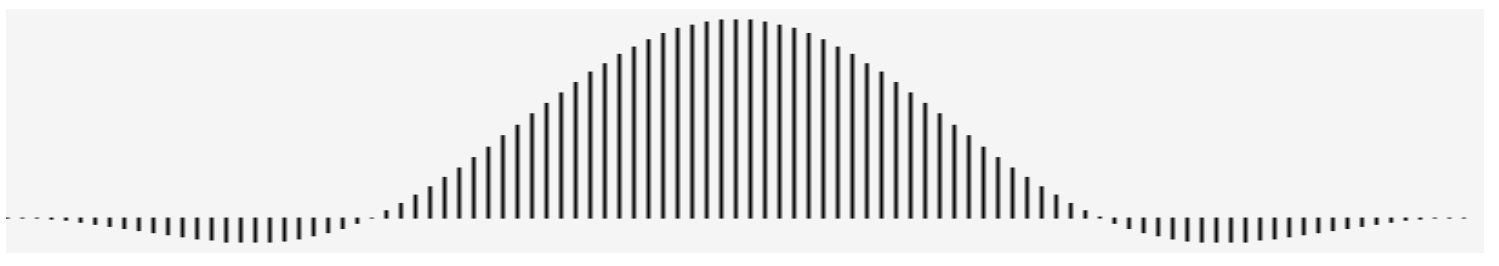

Fig. 1. The window used for filter synthesis. 
As can be seen in the following figure, the gravitational wave isolated in this way is frequency-limited from above, but has a minimum of out-of-band interference.

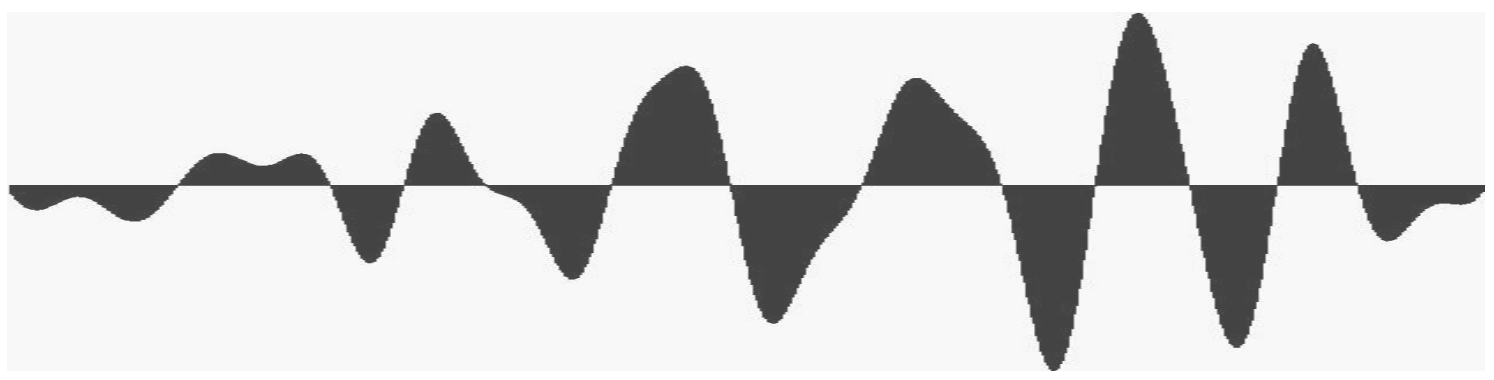

Fig. 2. Gravitational wave extracted from the original signal using a proprietary filter system. The original file H-H1_GWOSC 16KHZ_R1-1185389792-32.wav from the original LIGO data [10] contains the event GW170729 [11]. File after filtering: $\mathbf{H} 1$ filt.wav (listening to both records is possible).

Note that the original LIGO material (file H-H1_GWOSC 16KHZ_R1-118538979232.wav) has the signal spectrum shown in Figure 3. Of course, at such a signal-to-noise ratio it is necessary to suppress both dominant low-frequency components and high-frequency harmonics. The inevitable non-uniformity of the frequency response in the required bandwidth will be less detrimental for the subsequent analysis than the residual out-of-band components.
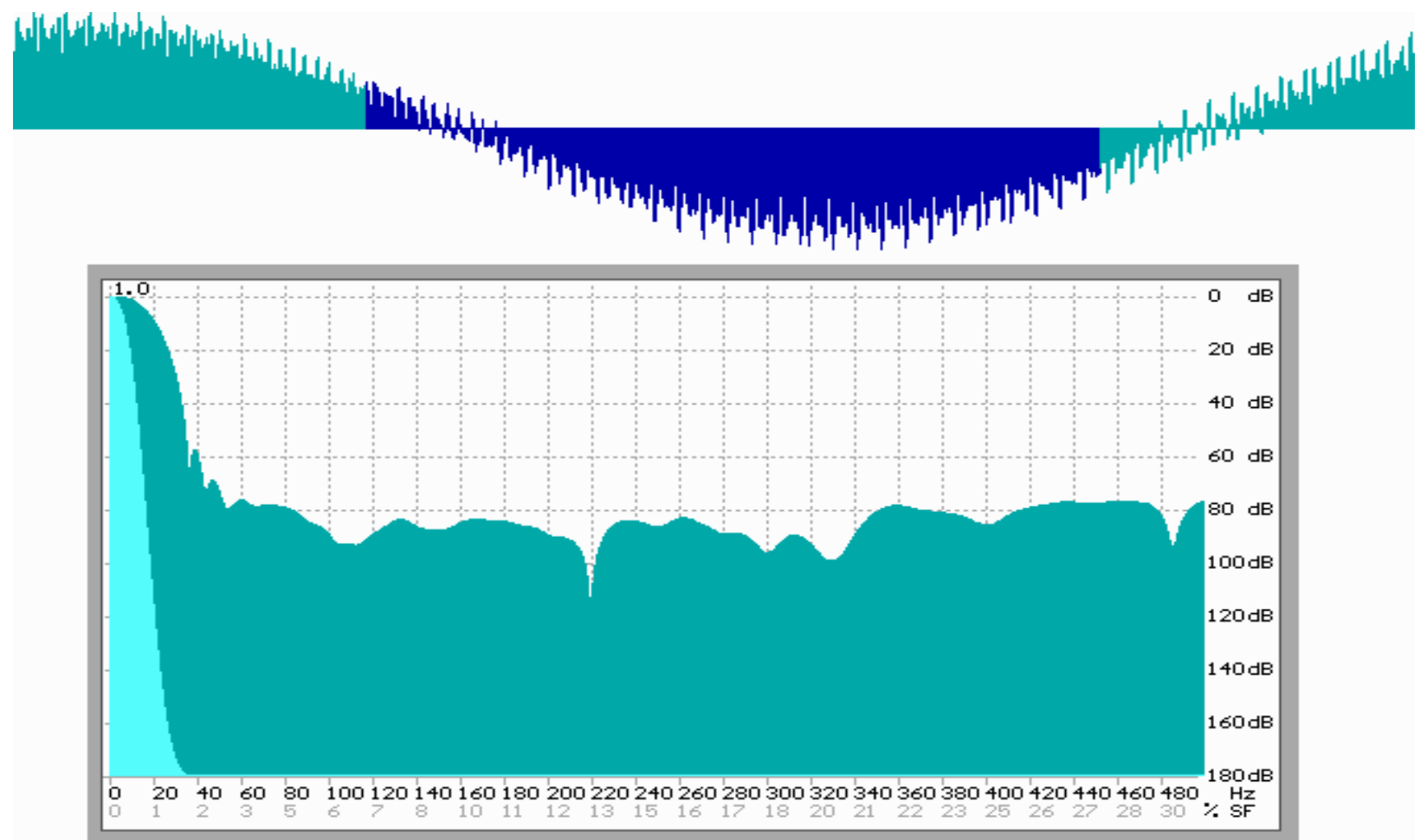

Fig. 3. Spectral estimation of the LIGO signal: at the top of the oscillogram (the area in which the gravitational wave of the event GW170729 was recorded is highlighted in blue [11]). At the bottom the energy spectrum, the X-axis scale is linear, the resolution is $0.5 \mathrm{~Hz}$. Hahn weighting window: green color is the spectrum in logarithmic scale by the ordinate, blue color shows it in linear one.

When filtering this signal the following results were obtained: $-200 \mathrm{~dB}(80+120)$ in the $0 \ldots .20 \mathrm{~Hz}$ band and at least $-126 \mathrm{~dB}$ at frequencies above $470 \mathrm{~Hz}$, which agrees well with the maximum possible suppression taking into account the residual digitization resolution.

The control data showed that the unevenness of the AFC in the range of $70 \ldots 250 \mathrm{~Hz}$ (i. e. in the least noisy GW range) did not exceed $6 \mathrm{~dB}$. 


\section{2 "Pure" gravitational wave pattern detection}

We can check the efficiency of the gravitational wave extraction and the correctness of the chosen filtering method by evaluating the operation of the convolutional detector. In our case we used as a prototype the time-inverted gravitational wave signal by the event GW170729 [11].
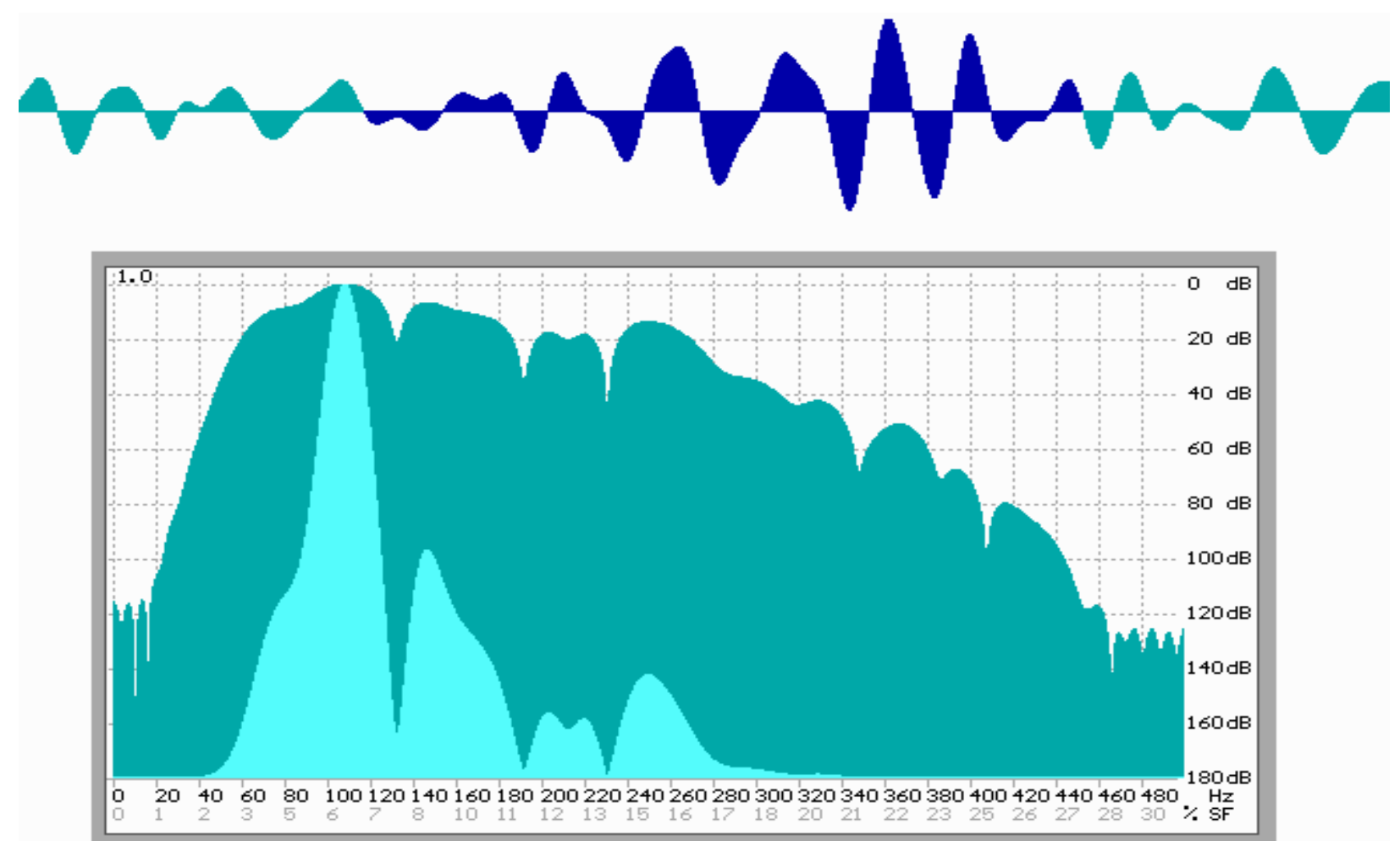

Fig. 4. Spectral estimation of the LIGO signal after FIR filtering: the signal is confidently separated from the noise, the suppression of out-of-band components is sufficient.

For each shift per sample of the signal of the original file H1_filt.wav two parameters has been calculated: a) the square of the Pearson correlation coefficient [ $\underline{3}$ ] and $b$ ) the current fragment of the convolved signal weighted by cosine window [12] $\omega_{i}$. The product of these parameters is presented as a graph unfolding synchronously with the original signal (see figure 5):

$$
V_{j}=\sum_{i=0}^{N-1}\left[X_{i} \frac{\left(1-\cos \frac{2 \pi i}{N-1}\right) \times\left(\sum_{k=0}^{N-1}\left(X_{k}-\bar{X}\right)\left(Y_{k}-\bar{Y}\right)\right)^{2}}{\sum_{k=0}^{N-1}\left(X_{k}-\bar{X}\right)^{2} \sum_{k=0}^{N-1}\left(Y_{k}-\bar{Y}\right)^{2}}\right]=\sum_{i=0}^{N-1} \frac{X_{i} \omega_{i}[\operatorname{cov}(X, Y)]^{2}}{s^{2}(X) \cdot s^{2}(Y)}
$$

where $V_{j}$ is the count of the output signal, $X$ is the realization of the input signal, $Y$ is the realization of the inverse prototype, $n$ is the length of realizations (in number of counts), $s^{2}$ is the variance of samples. The values of $V_{j}$ are always positive and will be the higher, the more the realization of $X$ correlates with the prototype of $Y$ and the higher the signal level of $X$.

\subsection{GW noise asymmetry detection}

To confirm the hypothesis about asymmetry of gravitational noise over time, it should be experimentally recorded and, if possible, measured.

Our basic idea is that (for a sufficiently long implementation) the statistically accumulated Pearson correlations of any time-symmetric signal (noise) with the direct and inverted prototypes will always be equal. Here, apparently, it is necessary to explain the idea by means of a simple model. So, let there is a digitized long Gaussian noise realization, and there is a short fragment of 


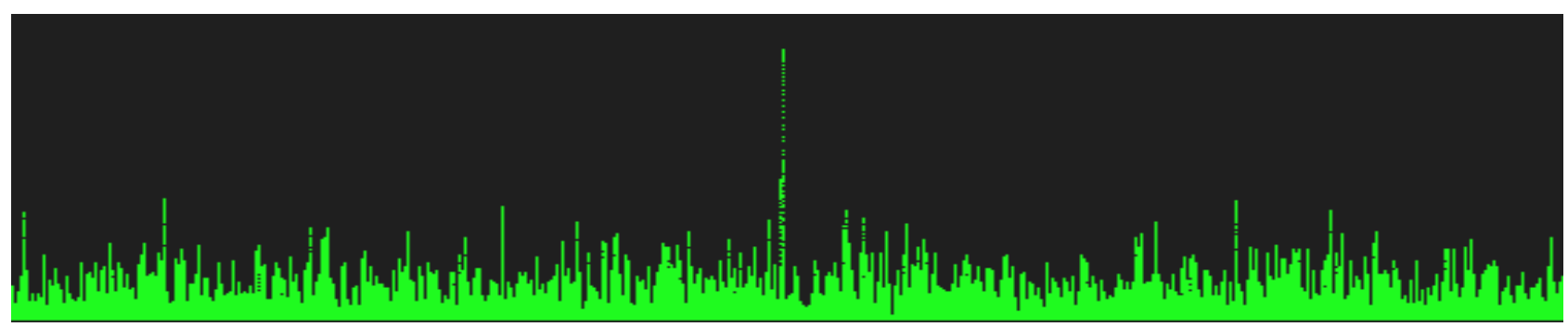

Fig. 5. Time series of $V_{j}$ values calculated by formula (2) for file $\mathbf{H 1}$ filt.wav. The duration of the recording fragment is 24 seconds. In the middle of the graph there is a response from the "officially confirmed" wave, at least three more bursts correspond to the fragments, about which there was a discussion about the correspondence of their form to the gravitational wave pattern.

asymmetric in time signal: for example, a digitized sound of Euler's falling disk [13] or an LFM radar pulse.

Now let this signal fragment slide along the noise realization. Calculating the Pearson coefficient at each step, we can make sure that at any time the value of process correlation will fluctuate between $-1.0<R<+1.0$. The accumulated sum of all coefficients will tend to zero, since the noise is not correlated with the signal.

If you reverse any of the signals in time, i. e. "backwards", and repeat the measurement, the result will be identical: the sum of the accumulated coefficients will tend to zero, and this is expected, since the noise is symmetrical in the time domain - it can be viewed from left to right or vice versa - nothing will change.

A completely different result will be obtained if the noise itself (NB!) consists of fragments of asymmetric in time signals. Suppose we have collected sounds of a very large number of simultaneously rotating and falling Euler disks. According to the central limit theorem, the total signal will be band-limited noise. Its statistical characteristics will be close to Gaussian (the closer, the more discs launched simultaneously). When calculating the correlation of this noise with a fragment of its generating signal, the following phenomenon must appear: the accumulated sum of correlations will depend on the direction of mutual displacement of the signals. And if at least one of signals is symmetric in time, there will be no asymmetry at accumulation of correlations, and the phenomenon will appear only if both (NB!) signals are asymmetric.

Let us perform processing of the real signals. To exclude the influence of signal amplitude on the result we will use only normalized correlation with the direct and inverse prototype. We will accumulate the difference of correlation coefficients for each sample of the signal and present it as a graph synchronous with the work of the detector, i. e.:

$$
\begin{aligned}
& R_{j}=R_{j-1}+\frac{\operatorname{cov}(X, Y)}{\sigma(X) \sigma(Y)}-\frac{\operatorname{cov}(X, Z)}{\sigma(X) \sigma(Z)}= \\
&=R_{j-1}+\left[\frac{\sum_{k=0}^{N-1}\left(X_{k}-\bar{X}\right)\left(Y_{k}-\bar{Y}\right)}{\sqrt{\sum_{k=0}^{N-1}\left(X_{k}-\bar{X}\right)^{2} \sum_{k=0}^{N-1}\left(Y_{k}-\bar{Y}\right)^{2}}}-\frac{\sum_{k=0}^{N-1}\left(X_{k}-\bar{X}\right)\left(Z_{k}-\bar{Z}\right)}{\sqrt{\sum_{k=0}^{N-1}\left(X_{k}-\bar{X}\right)^{2} \sum_{k=0}^{N-1}\left(Z_{k}-\bar{Z}\right)^{2}}}\right]
\end{aligned}
$$

where $X$ is the input signal array, $Y$ is the implementation of the inverse prototype, and $Z$ is the implementation of the non-inverse prototype.

Obviously, if the signals are statistically symmetric over time, the curve of time dependence $R$ will not deviate significantly either in the positive or in the negative region, i. e. accumulation will not occur. If the signal is asymmetric, the curve will shift monotonically up or down. 


\section{Results and discussion.}

As a zero reference, we first investigate the white normally distributed Gaussian noise that

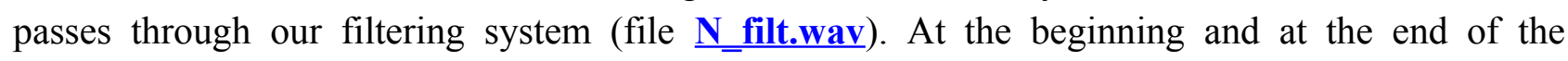
implementation, replace the noise with a monochromatic signal for clarity and convenience in evaluating the results (figure 6).

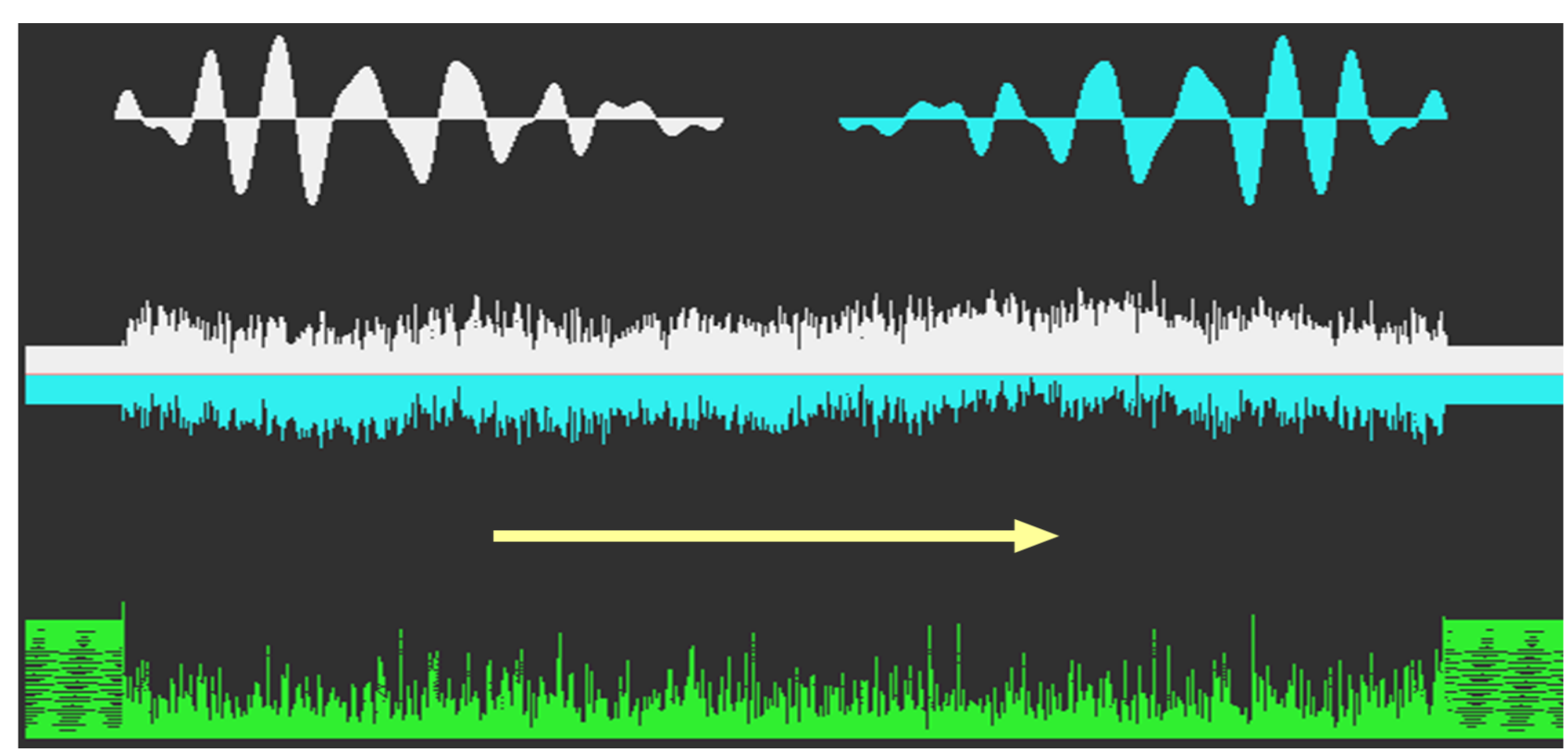

Fig. 6. Estimation of the asymmetry of the noise signal. At the top the prototypes with which the signal is convolved: light gray shows the time-inverted "direct" pattern Y, blue indicates the noninverted "inverse" pattern Z. In the middle is shown the graph of accumulation of difference $R_{j}$ of Pearson coefficients, i.e. the value of asymmetry. At the bottom, the time series $V_{j}$ is shown. The yellow arrow shows the direction of the time axis.

Note that during the observation time (30 s) there is no accumulated asymmetry, despite periodic small deviations in both directions. The same results were observed for all other noise, unmodulated harmonic signals, and any noise/signal combinations.

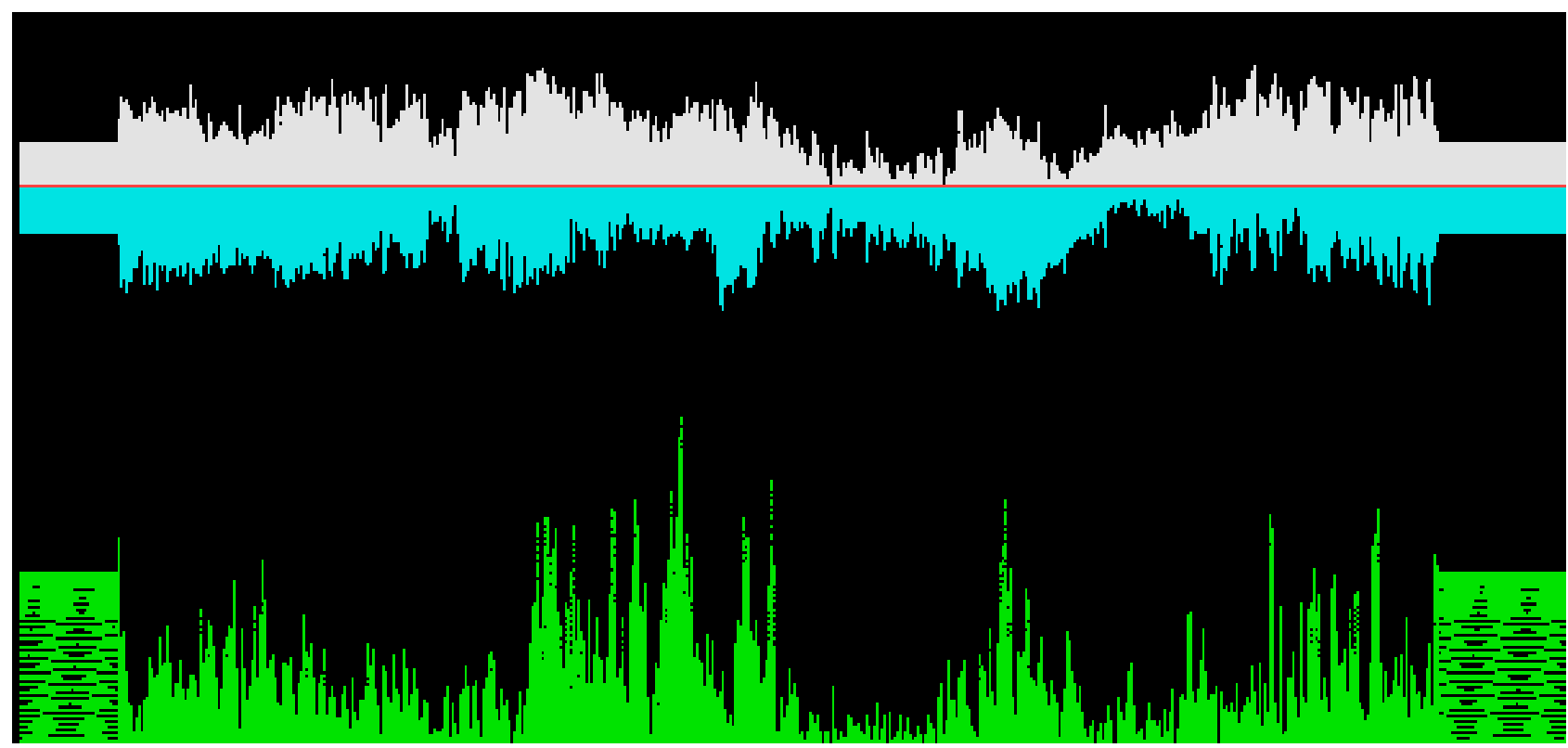

Fig. 7. Music signal analysis. Source file music.wav, file after filtering mus_filt.wav. Despite the fluctuations of the graph, the accumulated asymmetry is equal to zero. 
Completely different effects were observed in the analysis of real gravitational signals. For the Hanford Observatory, the shift of the correlation plot is confidently fixed. If we increase the observation time, the asymmetry will continue to grow monotonically (figure 8).

The vicinity of the most powerful gravitational wave is interesting with some anomaly lasting for units of seconds (the duration of the recording fragment is 30 seconds: the beginning and the end of the fragment are replaced by a monochromatic signal).

For the records from the Livingstone Observatory, the asymmetry is even larger (figure 9). Unfortunately, detector (2) does not record any obvious bursts, which is probably due to the prototype, which is isolated from the Hanford recordings-the signal shapes are apparently somewhat different.

The worst, but, nevertheless, comparable results are obtained for the Virgo records (figure 10). Unfortunately, the frequency drifting fan noise cannot be filtered out completely (the data of the compensation channels provided by the LIGO observatory [14] were not used in our work, since, unfortunately, they are not available for the event GW170729).

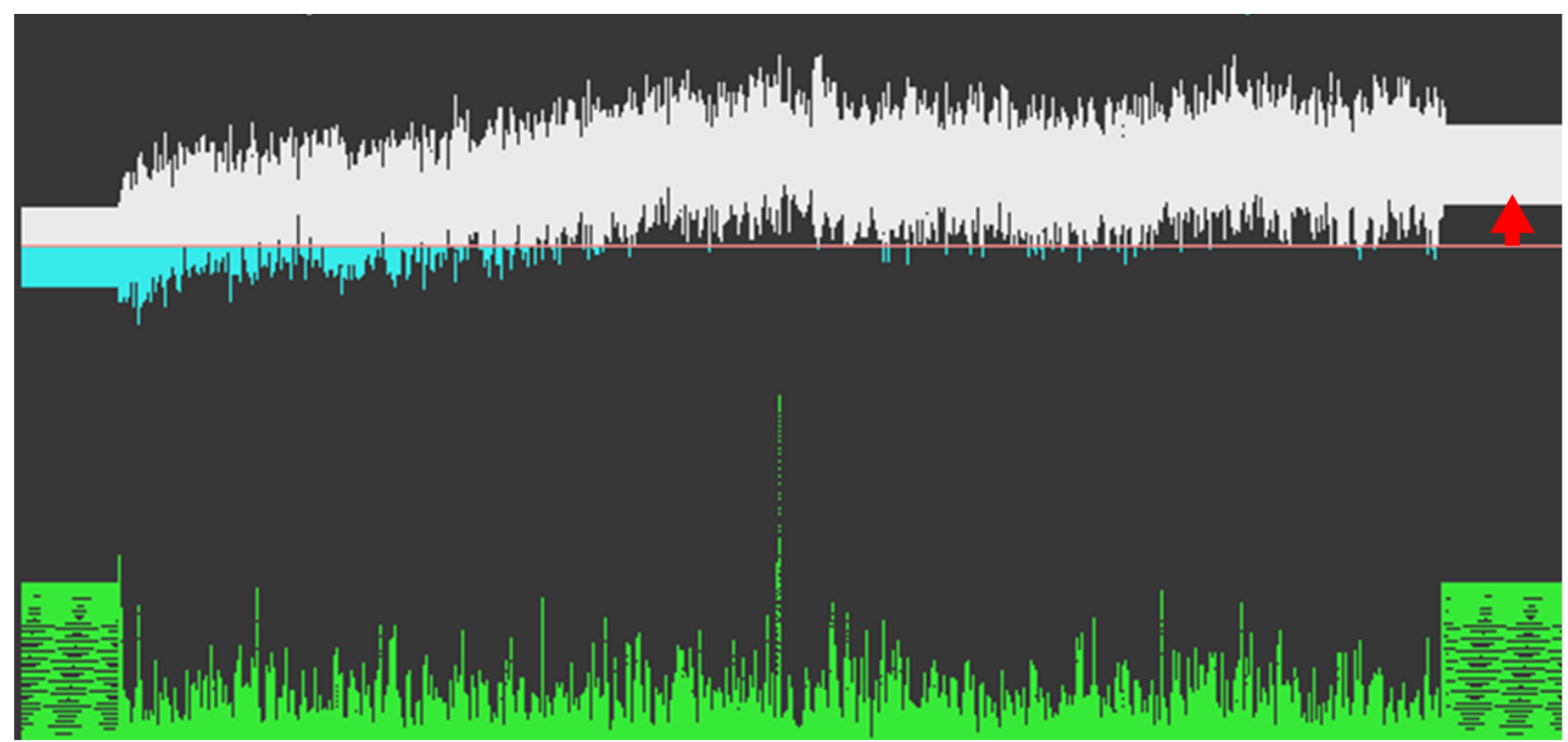

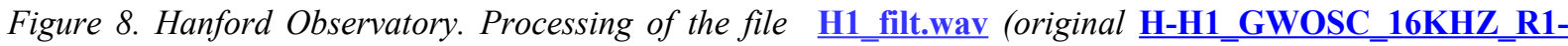
1185389792-32.wav). In contrast to the noise signals, the accumulated asymmetry is not equal to zero. The red arrow shows the rise of the plot.

It should be noted that for the LIGO recordings, the accumulated asymmetry is unequal: all signal registrations after hardware upgrades, i. e., reduction of intrinsic noise and improvement of the signal-to-noise ratio, give a more asymmetric signal (which is to be expected).

So, in all records of gravitational noise one observes asymmetry in time, i. e. the contribution of gravitational wave components is stably detected. But this is only a qualitative estimate. For quantitative estimates it is necessary to find some non-trivial method of measurement.

It is impossible in principle to separate hardware, seismic, thermal, Newtonian noise from gravitational noise. A basic statement of information theory states: "If signals exist simultaneously, and their spectra overlap, then complete separation of signals is impossible.

In our case, the only way to solve the problem remains: modeling signals with a known additive contribution of gravitational wave components to the structure of the Gaussian noise.

Let us make a measurement with a knownly high contribution of the "gravitational" component: let us add to the Gaussian noise fragments of the officially registered gravitational wave 


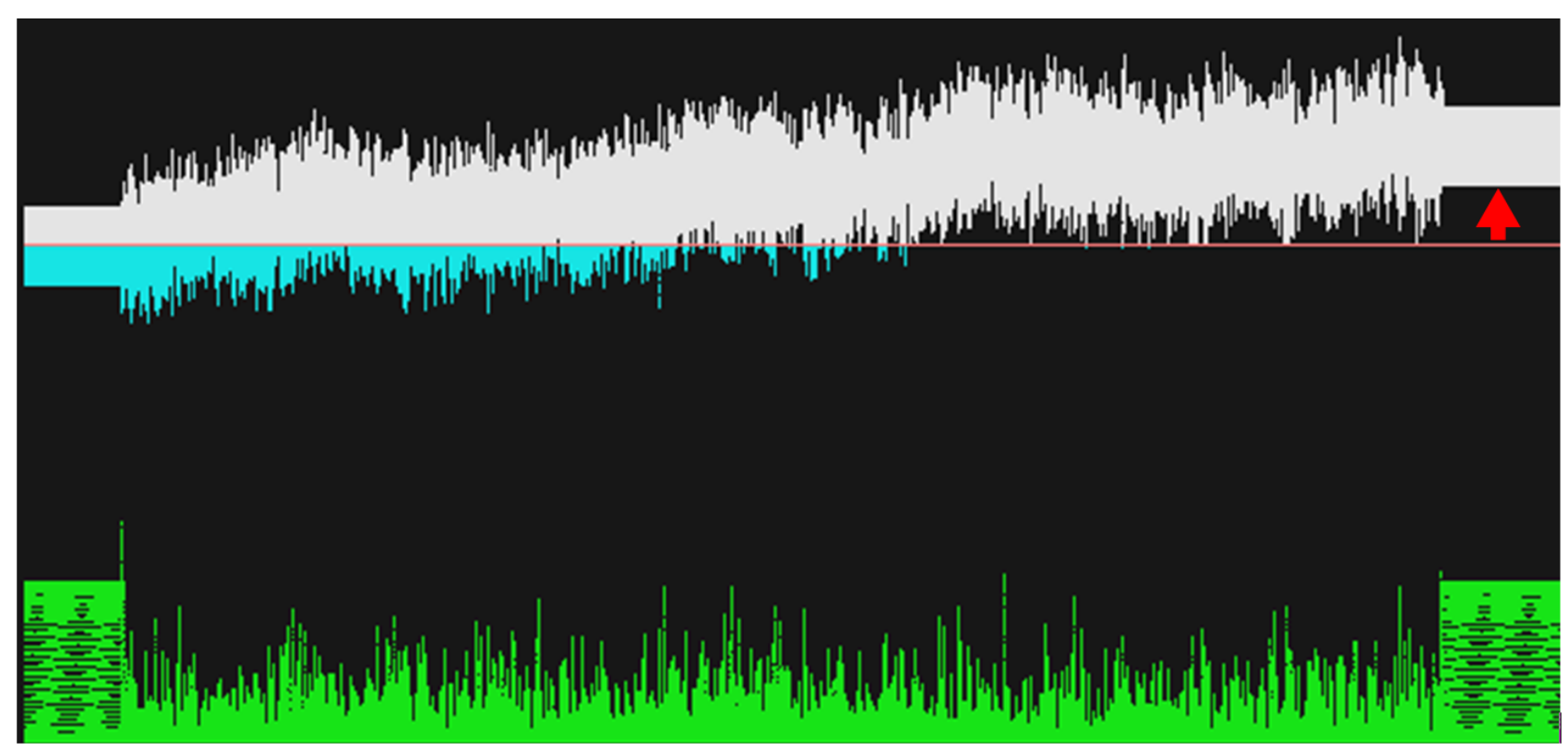

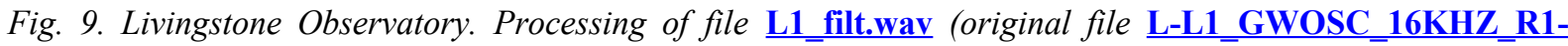
1185389792-32.wav). The asymmetry is clearly observed.

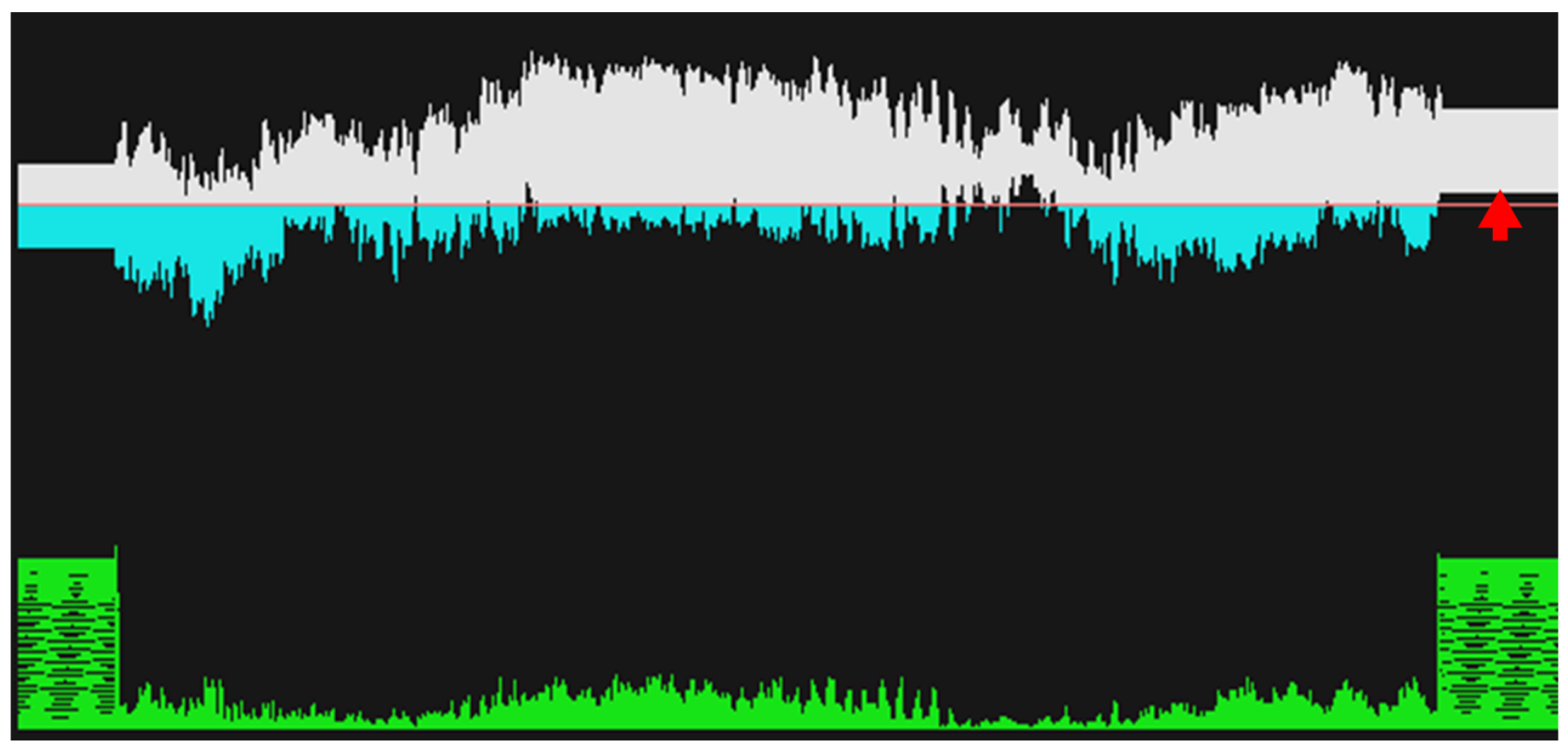

Fig. 10. Virgo Observatory. File V1_filt.wav (original V-V1_GWOSC_16KHZ_R1-1185389792-32.wav). Due to unsuppressed hardware interference, the detector does not work well. The signal asymmetry is unquestionable, but it is lower than in the previous illustrations.

so that the signal to noise ratio is $3: 1$, (figure 11 , at such a ratio the signal against the noise will already be observed visually).

Let us estimate the accumulation of asymmetry and monitor the detector performance (figure 12).

We will assume that the measuring instrument is pre-tested, and it is possible to select such a signal-to-noise ratio, which will allow to reach the values of the curve rise as in the cases of gravitational detector signal recordings.

Here we can add that partial separation of noise components is possible in the time domain, i. e. the signal is divided into two channels in a correlation way: asymmetric (left channel) and symmetric (right channel) noises. The stereo2.wav. file, when listened to, gives an idea of how the 


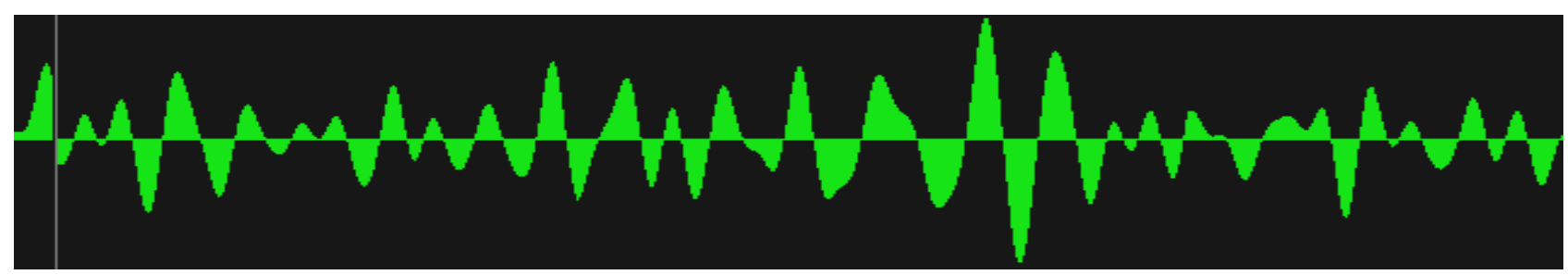

Fig. 11. Additive mixture of the components of gravitational waves and GWN in the ratio 3:1. As expected, the characteristic patterns are visually detectable.

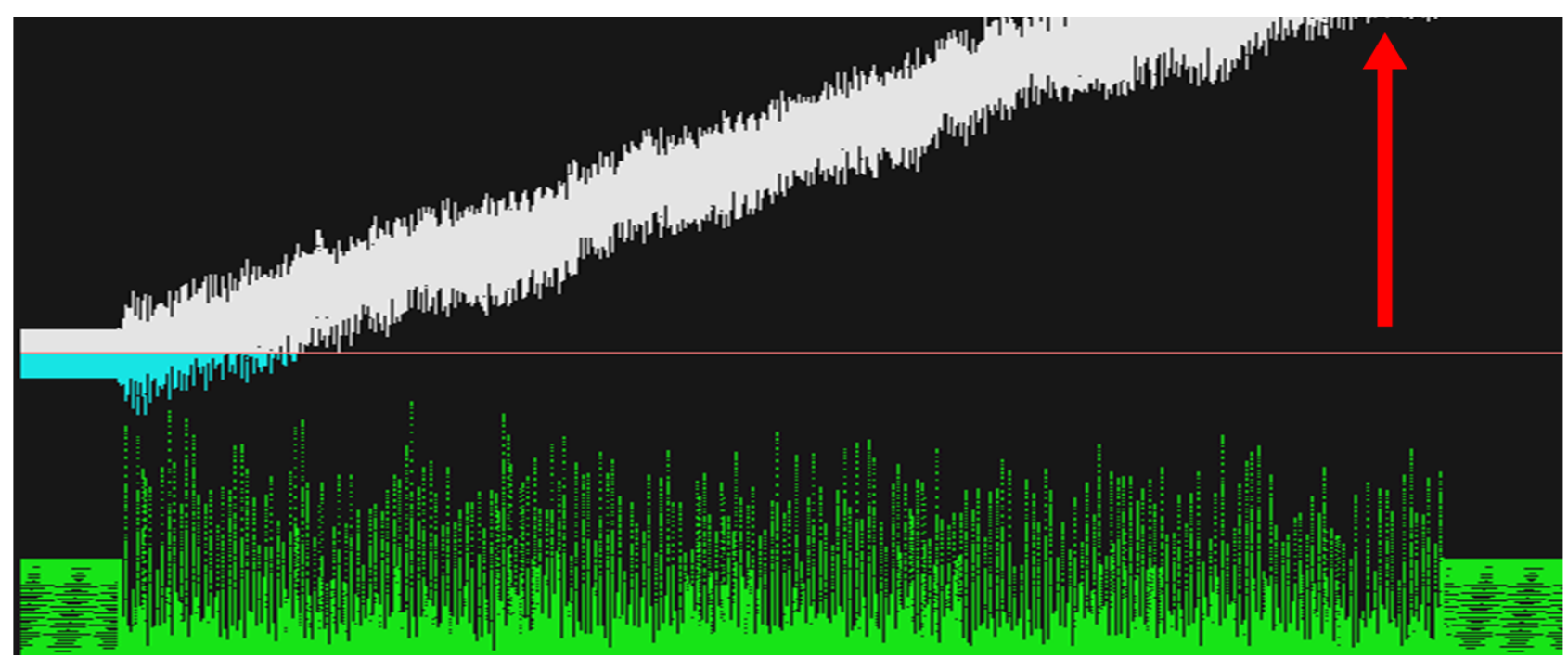

Fig. 12. Analyzer performance with a significant contribution of the "gravitational" component. The signal-tonoise ratio is 3:1. Multiple $V_{j}$ spikes are detected. The asymmetry is large, the accumulated difference of Pearson coefficients grows rapidly.

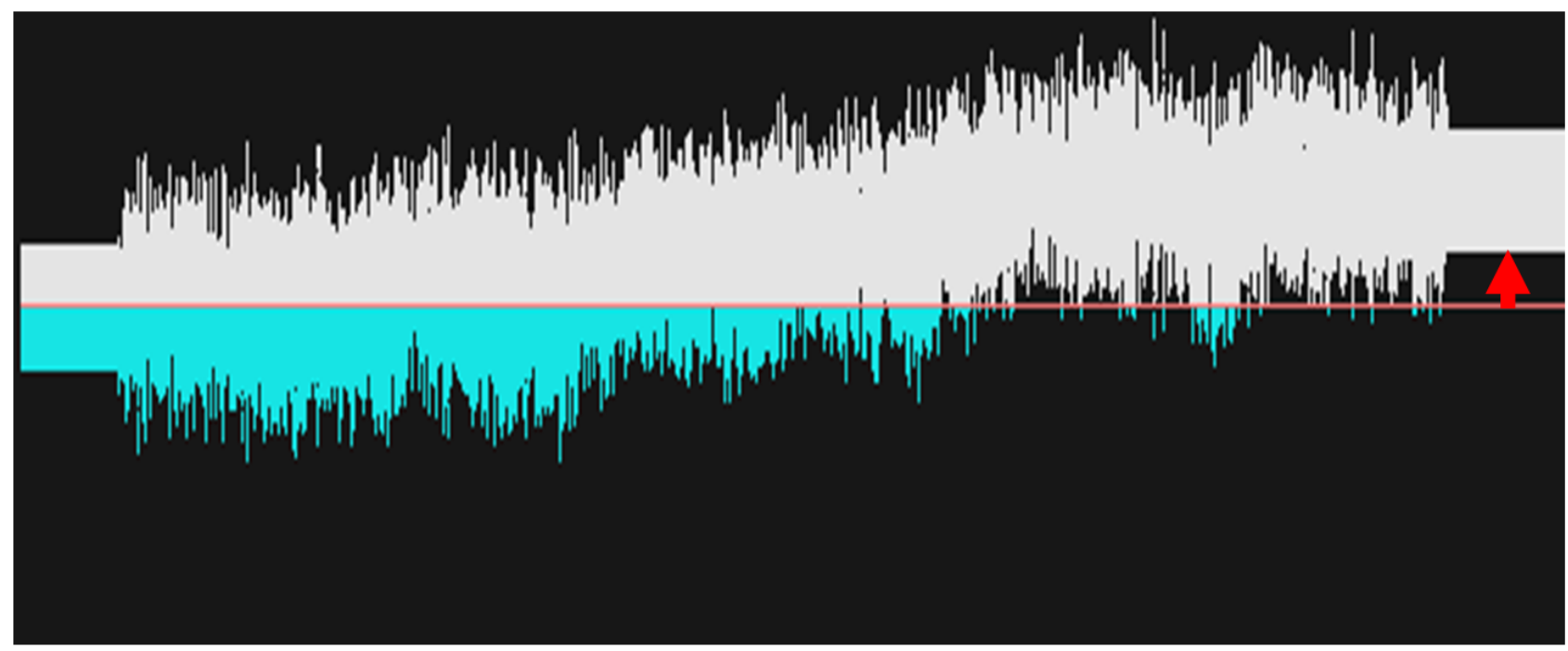

Fig. 13. The signal-to-noise ratio is 1:2. Complete correspondence to the average value of the asymmetry for the records of gravitational detectors. The estimate is, of course, preliminary.

purely noise and "gravitational" components differ (powerful pulses in the left channel at the 15th second correspond to the event GW170729)

\section{Conclusions}

So, the results of indirect measurements allow us to estimate that the gravitational noise at the output of the detectors is approximately $-6 \mathrm{~dB}$, and this, in fact, is an unexpectedly large value. It turns out that we should pay attention not only to the rarely observed high-amplitude gravitational 
waves, but also to the permanent noise, the essential part of which is formed by the same processes - merging of massive astronomical objects. The very presence of asymmetry of the signal received by detectors means that the whole space is filled with gravitational noise of sub-kilohertz range. And, consequently, any optical and radio-frequency signals always and everywhere propagate in the gravitational-noise field.

Then the input signal of any radio telescope directed to any point of space will include a ripple caused by gravitational noise and manifested as a differential modulation in the azimuth and angle of location in the picture plane of the observer (i. e. the change in the scattering ellipse and/or polarization).

Accordingly, at the output of the quadrature polarimeter with measurement time less than 1 ms and bandwidth limitation at frequencies below $70 \mathrm{~Hz}$ there will be an additive mixture of own noise and gravitational noise component, which can now be estimated (although it is still impossible to completely separate these noises). The contribution of gravitational noise may be small, but with increasing of realization, i.e. registration time, the accuracy of measurement will increase. Then maps of the gravitational noise of the sky can be obtained with the available radio telescopes. In addition, the relic radiation should also have traces of propagation in the gravitational noise field.

\section{References}

1. Bailes, M., Berger, B. K., Brady, P. R. et al. Gravitational-wave physics and astronomy in the 2020s and 2030s. Nat Rev Phys 3, 344-366 (2021). https://doi.org/10.1038/s42254-021$\underline{00303-8}$

2. Bendat, J. S. \& Piersol, A. G. (2010). Linear Physical Systems. In J.S. Bendat and A.G. Piersol (Eds.), Random Data (4th ed., pp. 25-44). John Wiley \& Sons, Inc. https://doi.org/10.1002/9781118032428.ch2

3. Bendat, J. S. \& Piersol, A. G. (2010). Stationary Random Processes. In J.S. Bendat and A.G. Piersol (Eds.), Random Data (4th ed., p. 116). John Wiley \& Sons, Inc. https://doi.org/10.1002/9781118032428.ch5

4. Larchenkova, T. I., Lutovinov, A. A., \& Lyskova, N. S. (2017). Influence of the galactic gravitational field on the positional accuracy of extragalactic sources. The Astrophysical Journal, 835(1), 51. https://doi.org/10.3847/1538-4357/835/1/51

5. Klauder, J. R., Price, A. C., Darlington, S., \& Albersheim, W. J. (1960). The Theory and Design of Chirp Radars. Bell System Technical Journal, 39(4), 745-808. https://doi.org/10.1002/j.1538-7305.1960.tb03942.x

6. Nikolaev, A. P., Krivonozhko, I. S., \& Sobkina, N. Y. (2018). Target travel compensation on long-term accumulation of radar signals. Journal of «Almaz - Antey» Air and Space Defence Corporation, 3, 12-19. https://doi.org/10.38013/2542-0542-2018-3-12-19

7. Трунин, Д. (2018, April 17). Физики научились измерять фоновый шум гравитационных волн в тысячу раз быстреe. Nplus1.Ru. https://nplus1.ru/news/2018/04/17/GW-noise

8. Smith, R., \& Thrane, E. (2018). Optimal Search for an Astrophysical Gravitational-Wave Background. Physical Review X, 8(2). https://doi.org/10.1103/physrevx.8.021019

9. Wilson, P. (2015). Chapter 9 - Digital Filters. In Design Recipes for FPGAs: Using Verilog and VHDL (2nd ed., pp. 117-134). Newnes.

10. GWOSC. (2020). Gravitational Wave Open Science Center. https://www.gw-openscience.org/eventapi/html/GWTC-1-confident/GW170729/v1/

11. Abbott, B., Abbott, R., Abbott, T., Abraham, S., Acernese, F., Ackley, K., Adams, C., Adhikari, R., Adya, V., Affeldt, C., Agathos, M., Agatsuma, K., Aggarwal, N., Aguiar, O., Aiello, L., 
Ain, A., Ajith, P., Allen, G., Allocca, A., . . Zweizig, J. (2019). GWTC-1: A GravitationalWave Transient Catalog of Compact Binary Mergers Observed by LIGO and Virgo during the First and Second Observing Runs. Physical Review X, 9(3). https://doi.org/10.1103/physrevx.9.031040

12. Barros, J., \& Diego, R. (2006). On the Use of the Hanning Window for Harmonic Analysis in the Standard Framework. IEEE Transactions on Power Delivery, 21(1), 538-539. https://doi.org/10.1109/tpwrd.2005.852339

13. Abbott, P., Keady, G., \& Tyler, S. (2010). Euler's disk: examples used in engineering and applied mathematics teaching. ANZIAM Journal, $51, \quad 360$. https://doi.org/10.21914/anziamj.v51i0.2596

14. GWOSC. (2021). Gravitational Wave Open Science Center. https://www.gw-openscience.org/auxiliary/GW170814/ 